\title{
Impact of Exclusive Breastfeeding on Digital Sucking in Children Attending the Children's Outpatient Department of University of Uyo Teaching Hospital, Uyo
}

\section{Oloyede Iso Precious ${ }^{\#}$ and Okpokowuruk Frances Sam ${ }^{\# *}$}

Department of Pediatrics, University of Uyo Teaching Hospital, Akwa Ibom State, Uyo, Nigeria

${ }^{\#}$ Both authors have contributed equally to this manuscript.

*Corresponding author: Dr. Frances Sam Okpokowuruk, Department of Pediatrics, University of Uyo Teaching Hospital, Akwa Ibom State, Uyo, Nigeria, Tel: +234-802-678-0888

\begin{abstract}
Background: Sucking of the digits is the most common oral habit found in children. It is a form of non-nutritive sucking which appears to be influenced by various factors. When the habit becomes prolonged, it can lead to both dental and hand complications. Breastfeeding is one of the factors known to be protective against this habit in children. Therefore, the aim of this study was to find out the prevalence of digital sucking in our environment and the influence of breastfeeding/exclusive breast feeding including various sociodemographic variables on this habit in children in our environment.
\end{abstract}

Methods: This study was a cross sectional, questionnaire-based study administered to one hundred and forty-five caregivers of children aged 0-18 years who attended the children's outpatient clinic of the hospital, consecutively recruited over a period of three months. Data was analysed using the Statistical Package for Social Science(SPSS) version 20.

Results: 145 respondents were analysed. The prevalence of digital sucking was $32.4 \%(95 \% \mathrm{Cl}=0.25-0.41)$ while the prevalence of exclusive breastfeeding was $47.6 \%$ (67 out of 144).

Exclusive breastfeeding and digit sucking demonstrated a significant negative association. Children who were exclusively breastfed were less likely to suck their fingers with a significant $p$-value of 0.025 -univariate analysis $(O R=2.28$; $95 \% \mathrm{Cl}=1.11-4.70)$. This finding was sustained on multivariate analysis with children who were exclusively breastfed having approximately a three times reduction in the odds of sucking their finger $(p=0.09 ; 95 \% \mathrm{Cl})$. There was no statistically significant difference $(p=0.252 ; \mathrm{OR}=0.96 ; 95 \% \mathrm{Cl}=$ $0.89-1.03$ ) between the mean total duration of breastfeeding for children who sucked their fingers compared with their siblings who did not suck their fingers $(9.73 \pm 4.90$ vs. 10.98 \pm 5.14 months). A history of both parents sucking had a positive association with digital sucking with a significant $p$-value of 0.036 (OR $=4.63 ; 95 \% \mathrm{Cl}=1.11$-19.40)-univariate analysis.

Conclusion: Prevalence of digital sucking in this study was $32.4 \%$ with children who were exclusively breastfed being less likely to suck their digits when compared to those who were not. Duration of breastfeeding beyond 6 months did not confer any additional advantage. A history of parental sucking was significantly associated with subsequent digital sucking in the children studied.

\section{Keywords}

Digital sucking, Exclusive breastfeeding, Sociodemographic factors

\section{Introduction}

Sucking of the digits is the most prevalent oral habit found in children [1]. Various digits are known to be involved in the sucking process with thumb sucking being the most common [1]. Digital sucking is a form of non-nutritive sucking and appears to be influenced by various factors such as age, sex, birth order, feeding method and socioeconomic status [2]. Breastfeeding is known to be associated with a lower frequency of non-nutritive sucking habits in children as opposed to bottle fed children and thus may be protective against the development of the habit with its attendant complications [3]. These complications can be divided into

Citation: Oloyede IP, Okpokowuruk FS (2018) Impact of Exclusive Breastfeeding on Digital Sucking in Children Attending the Children's Outpatient Department of University of Uyo Teaching Hospital, Uyo. Int J Pediatr Res 4:036. doi.org/10.23937/2469-5769/1510036

Accepted: September 10, 2018: Published: September 12, 2018

Copyright: (C) 2018 Oloyede IP, et al. This is an open-access article distributed under the terms of the Creative Commons Attribution License, which permits unrestricted use, distribution, and reproduction in any medium, provided the original author and source are credited. 
dental and hand complications. Hand complications include deformities that affect mainly the proximal and middle phalanges of the affected digits, a higher incidence of paronychia, herpetic whitlow, calluses and eczema [4]. Dental complications are mainly those that include malocclusions such as anterior open bite, posterior cross bite and excessive overjet [4]. The prevalence of digital sucking varies in different environments and different cultures and ranges between 1.7-47\% [2]. In our environment, very few studies have been done on this childhood habit, but prevalence rates have ranged between $7.2 \%-30.8 \%[5,6]$ respectively. Therefore, the rationale for this study was to find out the prevalence of digital sucking in children aged $0-18$ years attending the children's outpatient clinic and to elucidate the impact of exclusive breastfeeding and duration of breastfeeding if any on this habit in our environment.

\section{Study aim and objectives}

To determine the prevalence of digital sucking in children recruited consecutively over a three months period and elucidate the impact of exclusive breastfeeding and duration of breastfeeding on digital sucking.

1. Determine the prevalence of digital sucking among children in Uyo AKS.

2. Determine the prevalence of exclusive breastfeeding in children in Uyo AKS.

3. Determine the average duration of breastfeeding of children in Uyo AKS.

4. Compare the prevalence of exclusive breastfeeding between children who suck their fingers and those who do not.

5. Compare the total duration of breastfeeding between children who suck their fingers and those who do not.

\section{Methods}

This study was a cross sectional, observational and questionnaire-based study targeting parents who brought their children aged 0-18 years to the children outpatient (CHOP) clinic of the University of Uyo Teaching hospital.

Parents were recruited consecutively over a threemonth period until the calculated sample size was reached.

\section{Inclusion criteria}

1. Consent from the caregiver to answer the questions posed in the questionnaire.

2. All children (0-18 years) who sucked their finger for at least six months.

3. Study respondent is a biological parent of the child.

\section{Exclusion criteria}

Failure to answer both questions on exclusive breastfeeding and digital sucking.

\section{Sample size calculation}

The sample size was calculated using the Fischer formula [7]. Where $n=Z^{2} p q / d^{2}$.

Where $\mathrm{n}=$ minimum sample size; $\mathrm{Z}=$ confidence interval at $95 \%=1.96 ; \mathrm{P}=$ best estimate of the population prevalence $=9 \%[8]=0.09 ; q=$ complementary probability $(1-p)=0.91 ; d=$ degree of precision, set at $5 \%$. Thus, the ideal representative sample for the present study was calculated as

$$
\mathrm{n}=\left[(1.96)^{2} \times 0.09 \times 0.91\right] \div(0.05)^{2}=126
$$

$10 \%$ (13) was added for non-responders. Thus, the total minimum sample size was 139 .

\section{Questionnaire}

A semi-structured questionnaire, which included open and closed-ended questions, was administered to the children's caregivers to characterize the population, such as: sex, age, educational level and occupation of the respondent. Questions relating to the children included, presence of exclusive breastfeeding, age at onset of breastfeeding, total duration of breastfeeding, age at cessation of breastfeeding, presence of digital sucking habits, digit sucked, birth order of children with digital sucking, age at commencement of digital sucking, gender of child who sucked, interventions to stop non-nutritive sucking and outcome of intervention, history of non-nutritive sucking in the family and impression of the parents on the mode of inheritance. The questionnaire structure was based on the literature review and was adapted to the study's aims.

\section{Operational definitions}

1. Digital sucking: This involves placing the finger into the mouth and rhythmically repeating sucking contact for a prolonged duration of at least six months. This sucking action could be ongoing or in the past.

2. Index Parent sucking: A history of digital sucking in the study respondent.

3. Other parent sucking: A history of digital sucking in the partner or spouse of the respondent.

4. Impression of the parents on mode of inheritance: Parents impression on the mode of inheritance of digital sucking.

5. Main intervention to stop digital sucking: The main intervention instituted by the parents to stop the child from sucking his/her digits.

6. The following definitions of breastfeeding recommended by the World Health Organization (WHO) were employed [9].

Exclusive Breastfeeding (EBF): When the infant is fed the mother's milk directly or following expression, and 
no other liquid or solid food is administered, except for medicine drops or vitamins for the first six months of life.

Breastfeeding (BF): When the infant remains on both exclusive, predominant and nonexclusive breastfeeding, i.e., child is fed with either breast milk alone or associated with water or any type of supplement semisolid or solid or other milk.

\section{Data analysis}

Data was analysed using the statistical Package for the Social Science (SPSS) version 20. The prevalence of exclusive breastfeeding and digital sucking was determined. The odds ratio (OR) was used as the size measure in the bivariate analysis, with a 95\% confidence interval $(95 \% \mathrm{Cl})$. To test the correlation between the presence of non-nutritive sucking habits and the independent variables, both univariate and multivariate logistic regression was done, The Receiver Operator curve (ROC) was derived and $p$ values $\leq 0.05$ were considered to be significant.

\section{Results}

\section{Baseline characteristics of study participants and participant flow}

A total of 154 caregivers were enrolled in the study. Nine respondents were excluded from the study for failure to answer the questions on digital sucking and exclusive breastfeeding, therefore 145 respondents were analysed. The prevalence of digital sucking was $32.4 \%$ (95\% Cl $=0.25-0.41)$ while the prevalence of exclusive breastfeeding was $47.6 \%$ (67 out of 144). In addition, children who did not suck their fingers had a significantly higher $(p=0.02)$ prevalence of exclusive breastfeeding than children who sucked their finger $(40.79 \%$ vs. $23.19 \%)$. Furthermore, the median duration of breastfeeding for children in Uyo was 12 months (interquartile range of 6-14 months).

Table 1: Baseline characteristics of study respondents/participants.

\begin{tabular}{|l|l|}
\hline Variable & \multicolumn{2}{l|}{ Frequency (\%) } \\
\hline Caregivers age (years) $(\mathbf{n}=\mathbf{9 5})$ & $40(42.1)$ \\
\hline $21-30$ & $25(26.3)$ \\
\hline $31-38$ & $25(26.3)$ \\
\hline $39-46$ & $5(5.3)$ \\
\hline $47-54$ & \\
\hline Caregivers gender $(\mathbf{n}=\mathbf{1 0 0})$ & $23(23.0)$ \\
\hline Male & $77(77.0)$ \\
\hline Female & \\
\hline Caregivers educational level $\mathbf{( n = 7 9 )}$ & $3(3.8)$ \\
\hline Primary & $9(11.4)$ \\
\hline Secondary & $60(75.9)$ \\
\hline Tertiary & $7(8.9)$ \\
\hline Postgraduate & \\
\hline Caregivers occupation $\mathbf{( n = 9 5 )}$ & $73(76.8)$ \\
\hline Government employed & $12(12.6)$ \\
\hline Self-employed & $10(10.6)$ \\
\hline Unemployed & \\
\hline
\end{tabular}

Table 1 summarizes the demographic features of the study respondents. Among 145 caregivers, 23 (23\%) were males, while 77 (77\%) were females, Majority $(40 \%)$ of the caregivers were in the $21-30$ age range. In addition, majority $(75.9 \% ; 60$ out of 79$)$ of the caregivers had tertiary level of education. Most (76.8\%) of the caregivers were government employed.

Table 2 summarizes the socio-demographic characteristics, interventions and caregivers' inheritance perception of children who sucked their fingers. Majority (64.9\%; 24 out of 37 ) of the children who sucked their finger were male. In addition, first-born children (48.5\%; 16 out of 33 ) were more likely to suck their finger. Most children (42.5\%; 17 out of 40 ) commenced digital sucking by the first month of life. The commonest intervention (30.4\%; 14 out of 46 ) to stop digital sucking was manual removal, while in 11 (23.9\%) children, no intervention was instituted. Majority of the caregivers (51.7\%; 15 out of 29 ) reported that the interventions

Table 2: Socio-demographic characteristics, Interventions and Caregivers Inheritance perception of children who sucked their fingers.

\begin{tabular}{|c|c|c|}
\hline Variables & Frequency & Percentage (\%) \\
\hline \multicolumn{3}{|c|}{ Gender of the child $(n=37)$} \\
\hline Male & 24 & 64.9 \\
\hline Female & 13 & 35.1 \\
\hline \multicolumn{3}{|l|}{ Birth order $(n=33)$} \\
\hline 1 & 16 & 48.5 \\
\hline 2 & 11 & 33.3 \\
\hline 3 & 4 & 12.1 \\
\hline 4 & 2 & 6.1 \\
\hline \multicolumn{3}{|c|}{ Age at commencement of digital sucking $(n=40)$} \\
\hline 1 month & 17 & 42.5 \\
\hline 2-3 months & 12 & 30 \\
\hline 3-4 months & 11 & 27.5 \\
\hline \multicolumn{3}{|l|}{ Interventions $(n=46)$} \\
\hline Wrapping of finger & 8 & 17.4 \\
\hline Manual removal & 14 & 30.4 \\
\hline $\begin{array}{l}\text { Application of bitter } \\
\text { substance }\end{array}$ & 6 & 13.1 \\
\hline Verbal reprimand & 4 & 8.7 \\
\hline Nothing & 11 & 23.9 \\
\hline Physical punishment & 3 & 6.5 \\
\hline \multicolumn{3}{|c|}{ Effectiveness in intervention $(n=29)$} \\
\hline Yes & 15 & 51.7 \\
\hline No & 14 & 48.3 \\
\hline \multicolumn{3}{|c|}{ Caregivers inheritance perception $(n=45)$} \\
\hline \multicolumn{3}{|c|}{ Index parent sucked } \\
\hline Yes & 5 & 11.1 \\
\hline No & 27 & 60 \\
\hline Don’t know & 13 & 28.9 \\
\hline \multicolumn{3}{|l|}{ Other parent sucked } \\
\hline Yes & 5 & 11.1 \\
\hline No & 22 & 48.9 \\
\hline Don’t know & 18 & 40 \\
\hline \multicolumn{3}{|l|}{ Runs in family } \\
\hline Yes & 4 & 8.9 \\
\hline No & 26 & 57.8 \\
\hline Don't know & 15 & 33.3 \\
\hline
\end{tabular}


were effective. In addition, majority of the caregivers did not think that digital sucking had a familial inheritance.

\section{Comparison of children who sucked their finger and those who did not suck their finger}

Table 3 shows the association (univariate analysis) between exclusive breastfeeding, total duration of breastfeeding, number of children in the family, history of parental sucking and digital sucking. There was a negative association between exclusive breastfeeding and

Table 3: Association between exclusive breastfeeding, total duration of breastfeeding, number of children in the family, history of parental sucking and digital sucking (univariate analysis).

\begin{tabular}{|l|l|l|l|}
\hline Variable & p-value & Odds ratio & $\mathbf{9 5 \%} \mathbf{C l}$ \\
\hline Exclusive BF & 0.025 & 2.28 & $1.11-4.70$ \\
\hline Total duration of BF & 0.252 & 0.96 & $0.89-1.03$ \\
\hline Index parent sucked & 0.010 & 0.30 & $0.12-0.75$ \\
\hline Other parent sucked & 0.019 & 0.39 & $0.18-0.86$ \\
\hline Both parents sucked & 0.036 & 4.63 & $1.11-19.4$ \\
\hline Number of children & 0.560 & 1.09 & $0.81-1.46$ \\
\hline
\end{tabular}

*Breastfeeding.

Table 4: Association between exclusive breastfeeding, total duration of breastfeeding, number of children in the family, history of both parental sucking and digital sucking (multivariate analysis) $(n=97)$.

\begin{tabular}{|l|l|l|l|}
\hline Variable & p-value & Odds ratio & $\mathbf{9 5 \%} \mathbf{C l}$ \\
\hline Exclusive $\mathrm{BF}^{*}$ & 0.009 & 3.19 & $1.33-7.64$ \\
\hline Total duration of BF & 0.463 & 0.97 & $0.89-1.05$ \\
\hline Both parents sucked & 0.238 & 2.66 & $0.52-13.51$ \\
\hline Number of children & 0.418 & 1.16 & $0.81-1.66$ \\
\hline
\end{tabular}

*Breastfeeding. digital sucking. Children who were exclusively breastfed were less likely to suck their fingers with a significant p-value of $0.025(\mathrm{OR}=2.28 ; 95 \% \mathrm{Cl}=1.11-4.70)$.

There was a significantly positive association of a history of parental sucking and digital sucking. A history of both parents sucking had a positive association with digital sucking with a significant $p$-value of $0.036(O R=4.63$; $95 \% \mathrm{Cl}=1.11-19.40)$. However, there was no significant association between total duration of breastfeeding and number of children in the family with digital sucking ( $p=0.252$ and 0.560 respectively). Furthermore, there was no statistically significant difference $(p=0.252$; OR $=0.96 ; 95 \% \mathrm{Cl}=0.89-1.03$ ) between the mean total duration of breastfeeding for children who sucked their fingers compared with their siblings who did not suck their fingers ( $9.73 \pm 4.90$ vs. $10.98 \pm 5.14$ months).

Table 4 shows the association (multivariate analysis) between exclusive breastfeeding, total duration of breastfeeding, number of children in the family, history of both parental sucking and digital sucking. (multivariate analysis). A binary logistic regression analysis including other important variables showed that exclusive breastfeeding was the only variable that was significantly associated with digital sucking. Children who were exclusively breastfed had approximately three times reduction in the odds of sucking their finger $(p=$ 0.09; 95\% Cl).

A ROC constructed to determine the sensitivity and specificity of exclusive breastfeeding on the reduction of digital sucking in children had an AUC of 0.67 (Figure 1).

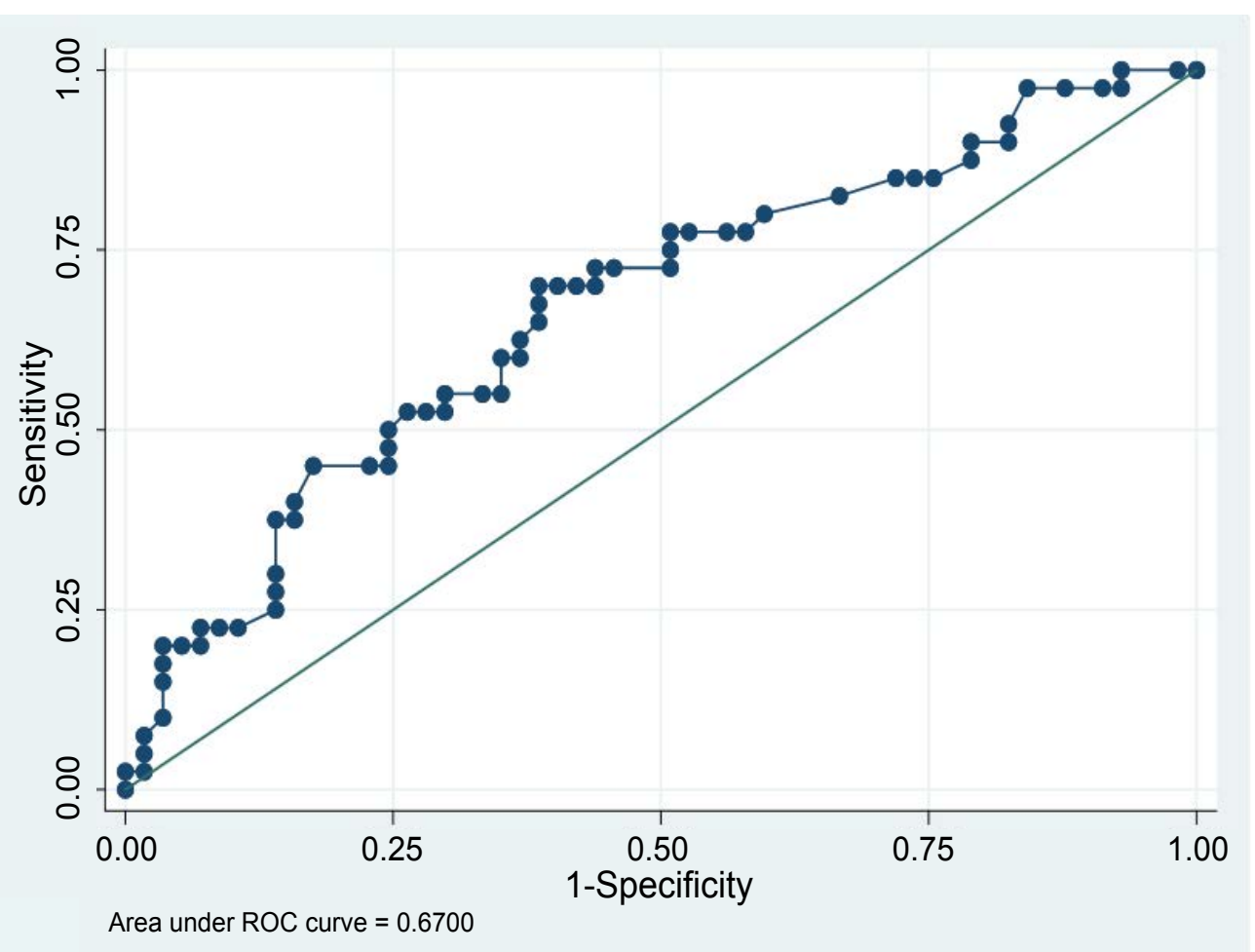

Figure 1: ROC curve showing the association between exclusive breastfeeding and Digital sucking. 


\section{Discussion}

The act of suckling is a normal physiological reflex found in infants and young children and is associated with the satisfaction of nutritional and emotional needs [10] however, persistence of this habit beyond the age of 48 months is considered abnormal and gradually becomes associated with dental and digital complications [4]. The overall prevalence of digital sucking in this study was $32.4 \%$. The higher prevalence of digit sucking found in this study could be explained by the fact that our study included children who were aged 0-18 years, and either were currently engaged in sucking their digits or had sucked in the past. It was also similar to findings by lbekwe, et al. among Ibo infants and Chopra, et al. in India who recorded high prevalence rates of $30.8 \%$ and $25 \%$ in children aged $0-11$ years respectively $[1,11]$. This is contrary to findings by Kolawole and Quashie-Williams in south western Nigeria who obtained prevalence rates of $17 \%$ and $7.2 \%$ respectively $[5,6]$. This variation in prevalence rates by different researchers is very likely due to differences in study design such as the age groups studied, type of study and sampling methods [12]. Some authors have noted that the prevalence of digital sucking decreases as the age increases [13] while others have not $[11,14]$.

The prevalence of exclusive breastfeeding in this study was $47.6 \%$ which is much higher than the national rate of $23.7 \%$ and in particular, the rate for Akwa Ibom state of $24.9 \%$ [15]. The higher rate obtained in this study may be because the study was not only hospital based but respondents were majorly resident in the urban area where awareness and education are much more prevalent as observed in the study population where $75 \%$ of caregivers had tertiary level of education. In this study, infants who were exclusively breastfed were 2-3 times less likely to suck their fingers when compared to those who were not. This is in keeping with findings by various authors $[3,8,14,16]$. Suckling is an innate urge that is very strong in intrauterine life and in infancy, which is satisfied when the child breastfeeds because of the exertion of the buccal muscles. The psychologi$\mathrm{cal} / \mathrm{emotional}$ satisfaction derived by these infants who are exclusively breastfed is not present in those who do not breastfeed and thus they look for substitutes such as digit sucking to satisfy their suckling urge [16]. This is the likely explanation for this "protective effect" of exclusive breastfeeding as seen in this study and others.

Duration of breastfeeding beyond six months of age was not found to confer any additional advantage in this study. This is contrary to the findings by Alburquerque, et al. and Yonezu who demonstrated that longer breastfeeding duration reduced the frequency of digit sucking $[3,17]$.

The attitude of mothers/caregivers to digital sucking varies from population to population and may depend on several factors such as the culture prevalent in the area, awareness, education and socioeconomic status [18]. In the index study, the prevalent attitude among caregivers was to institute one form of intervention or the other ranging from manual removal of the finger, wrapping of the finger, application of a bitter substance, verbal reprimand and physical punishment with over half the respondents reporting that whatever interventions were instituted were effective. This is similar to findings by Al Hussyeen in Saudi Arabia where mothers also intervened though a high percentage reported that their interventions were ineffective especially in children who practiced digit sucking as opposed to findings in this study [18]. The attitude of mothers in India differed significantly from what obtained in this study and that in Saudi mothers as sixty eight percent of them did not attempt to use any measures to stop their children from digit sucking [1].

Although majority of the caregivers did not think that digital sucking had a familial inheritance, in this study, a history of parental sucking (either one or both parents) as a child was found to have a weak association with digital sucking in the children studied. Some studies have proposed that behavioral genetics may have a role to play in non-nutritive sucking especially in those in whom the behavior is prolonged [19]. Another study done by Oooki [12] on over one thousand twin pairs (both monozygotic and dizygotic) in Japan suggests a strongly genetic component to digit sucking which has been highlighted in this study and warrants further investigation.

Other researchers $[1,16,18,20]$ have studied the relationship between sociodemographic factors such as parental age, education, occupation, family size and family income with digital sucking howbeit, this was not the aim of this study but can serve as a focus for future research.

The limitations of this study include recall bias as some parents could not remember some details about their children's habit leading to a lot of missing data. Secondly, gender was not included in bivariate and multivariate analysis since gender was only recorded for children who sucked their finger. Lastly, the small sample size of the index study makes the findings preliminary which would warrant a larger study in the future. Despite the limitations of this study, it has shown another protective effect of exclusive breastfeeding. It is also likely the first study of its kind in Nigeria that demonstrates the impact of inheritance on digital sucking although because of the small sample size, the results should be interpreted with caution and further studies with larger numbers and genetic studies are needed to fully assess this effect of genetics.

\section{Conclusion}

Digit sucking was found to be prevalent in about one third of the study participants with exclusive breastfeed- 
ing acting as the single most important factor in helping to protect children from acquiring this habit. Over eighty percent of the mothers/caregivers in this study instituted one form of intervention or another indicating a low acceptance of the habit in their children. Although the parents of children who sucked did not believe that digit sucking had a familial basis, the findings in this study are to the contrary and merits further research.

\section{References}

1. Chopra A, Lakhanpal M, Singh V, Gupta N, Rao NC, et al. (2015) The habit of digit sucking among children and the attitude of mothers towards the habit in India. TMU J Dent 2: $1-4$.

2. Srinivasan D, Aegappan AR, Joe Louis C (2012) Management of persistent non nutritive sucking habit. Chettinad Health City Med J 1: 62-64.

3. de Albuquerque SSL, Duarte RC, Cavalcanti AL, Beltrão E de $M(2010)$ The influence of feeding methods in the development of nonnutritive sucking habits in childhood. Cienc Saude Coletiva 15: 371-378.

4. Davidson $L$ (2008) Thumb and finger sucking. Pediatr Rev 29: 207-208.

5. Kolawole KA, Folayan MO, Agbaje HO, Oyedele TA, Oziegbe EO, et al. (2016) Digit sucking habit and association with dental caries and oral hygiene status of children aged 6 months to 12 years resident in semi-urban Nigeria. PLoS One 11: e0148322.

6. Quashie-Williams R, daCosta OO, Isiekwe MC (2010) Oral habits, prevalence and effects on occlusion of 4-15 year old school children in Lagos, Nigeria. Niger Postgrad Med J 17: 113-117.

7. Araoye MO (2003) Subject selection and sample size determination. In: Research methodology with statistics for health and social sciences. ( $4^{\text {th }}$ edn), Nathadex, Ilorin, Nigeria, 115-120.
8. Lopes T, de Deus Moura L, Lima MC (2014) Breastfeeding and sucking habits in children enrolled in a mother-child health program. BMC Res Notes 7: 362.

9. World Health Organization (1991) Indicators for assessing breast-feeding practices.

10. Johnson ED, Larson BE (1993) Thumb-sucking: Classification and treatment. ASDC J Dent Child 60: 392-398.

11. Ibekwe R, Ibeziako N, Ibe B, Ibekwe M (2011) Digit-sucking among Ibo infants in Eastern Nigeria. J Paediatr 38: 73-77.

12. Ooki S (2005) Genetic and environmental influences on finger-sucking and nail-biting in Japanese twin children. Twin Res Hum Genet 8: 320-327.

13. Magalhães L do NC, Rodrigues MJ, Heimer MV, Alencar AS de (2012) Prevalence of non-nutritive sucking habits and its relation with anterior open bite in children seen in the odontopediatric clinic of the University of Pernambuco. Dent Press J Orthod 17: 119-123.

14. Orimadegun AE, Obokon GO (2015) Prevalence of non-nutritive sucking habits and potential influencing factors among children in urban communities in Nigeria. Front Pediatr 3: 30.

15. UNICEF (2018) Infant and young child feeding-UNICEF data.

16. Moimaz SAS, Rocha NB, Garbin AJI, Saliba O (2011) Relationship between breastfeeding and non-nutritive sucking habits. Ciênc Saúde Coletiva 16: 2477-2484.

17. Yonezu T, Arano-Kojima T, Kumazawa K, Shintani S (2013) Association between feeding methods and sucking habits: A cross-sectional study of infants in their first 18 months of life. Bull Tokyo Dent Coll 54: 215-221.

18. Al-Hussyeen AJA (2010) Attitudes of Saudi mothers towards prolonged non-nutritive sucking habits in children. Saudi Dent J 22: 77-82.

19. Hatala J (2017) Behavioral genetics and thumb sucking in adolescents. Dent 3000.

20. Rajchanovska Domnika, Ivanovska Beti Zafirova (2014) Prevalence of finger sucking at preschool children in Bitola. Int J Sci Appl Res 13: 292-302. 